\title{
Selective Contribution of Waist Circumference Reduction on the Improvement of Sleep-disordered Breathing in Patients Hospitalized with Type 2 Diabetes Mellitus
}

\author{
Susumu Kashine ${ }^{1}$, Ken Kishida ${ }^{1,2}$, Tohru Funahashi ${ }^{1,2}$, Tetsuyuki Yasuda ${ }^{1}$, Kohei Okita ${ }^{1}$, \\ Yuji Matsuzawa $^{3}$ and Iichiro Shimomura ${ }^{1}$
}

\begin{abstract}
Objective Sleep-disordered breathing (SDB) is a potential risk factor for cardiac sudden death. Recent studies have reported that patients with type 2 diabetes mellitus (T2DM) frequently suffer from SDB. Although the roles of hyperglycemia, disturbances of the autonomic nervous system and obesity have been postulated, the factors related to SDB in T2DM, especially those related to improvement of SDB remain unknown. We investigated the significance of waist circumference (WC), representing excess visceral fat, body mass index (BMI), glycemic control and other clinical parameters on SDB in T2DM.

Methods and Subjects Forty inpatients received treatment for T2DM. Overnight cardiorespiratory monitoring and laboratory tests were conducted before and after treatment of T2DM.

Results The apnea-hypopnea index (AHI) at admission correlated positively with BMI, neck circumference, WC, and systolic and diastolic blood pressures, but not with Log 1,5-anhydro-D-glucitol (1,5-AG) and presence or absence of diabetic neuropathy. Stepwise multiple regression analysis identified BMI and WC as significant determinants of AHI. After 2 or 3 weeks of glucose-lowering therapy, hyperglycemia was controlled and significant reductions in AHI, BMI, WC, 1,5-AG, leptin, high-sensitivity C-reactive protein (hs-CRP), and an oxidative stress marker, thiobarbituric acid reactive substances (TBARS) were observed. The fall in AHI correlated significantly with changes in WC independent of BMI, 1,5-AG, leptin, hs-CRP, and TBARS. Conclusion Our results demonstrated that reduction of WC correlated with improvement in SDB independent of glycemic control in T2DM, and that abdominal obesity might be a target for the treatment of SDB and prevention of potential cardiovascular diseases in T2DM.
\end{abstract}

Key words: sleep-disordered breathing, type 2 diabetes mellitus, waist circumference reduction, adipocytokines

(Intern Med 50: 1895-1903, 2011)

(DOI: 10.2169/internalmedicine.50.5669)

\section{Introduction}

Sleep-disordered breathing (SDB), especially the sleep apnea-hypopnea syndrome, is a risk factor for traffic accidents (1), sudden death (2) and atherosclerotic cardiovascular events (3). Recent work has also demonstrated that SDB is frequently associated with type 2 diabetes mellitus (T2DM) (4-12). Therefore, the diagnosis and treatment of
SDB are important in order to prevent cardiac death in T2DM.

In SDB, activation of the sympathetic nervous system, stress associated with repetitive hypoxemia, production of reactive oxygen species, and inflammatory and thrombotic processes are postulated as potential mechanisms of atherosclerotic vascular changes (13-15). Furthermore, these processes are hypothesized to worsen insulin resistance (16). However, the etiological factors involved in the development

${ }^{1}$ Department of Metabolic Medicine, Osaka University, Japan, ${ }^{2}$ Department of Metabolism and Atherosclerosis, Graduate School of Medicine, Osaka University, Japan and ${ }^{3}$ Sumitomo Hospital, Japan

Received for publication April 18, 2011; Accepted for publication May 24, 2011

Correspondence to Dr. Ken Kishida, kkishida@imed2.med.osaka-u.ac.jp 
of SDB in T2DM remain unclear. Profound hyperglycemia, dysregulation of the autonomic nervous system and the presence of obesity are postulated.

In obese patients, SDB is related to visceral obesity rather than subcutaneous obesity (17-19). Excess visceral fat may restrict diaphragm dynamics during sleep and forced breathing may result in collapse of the airway. Excess visceral fat also equates with adipocyte dysfunction (20). The inflammatory changes and oxidative stress in adipose tissue trigger dysregulation of adipocytokines, which enhance insulin resistance and atherosclerotic vascular changes (21). Recently, we found that circulating levels of adiponectin were low and further decreased during sleep in patients with severe obstructive sleep apnea-hypopnea syndrome and abdominal obesity (22), in part due to hypoxic stress in visceral adipose tissue compared with subcutaneous adipose tissue (23). The association of SDB with visceral fat accumulation may accelerate these processes.

The average body mass index (BMI) of Japanese patients with T2DM is lower than in Caucasians (24). The number of T2DM Japanese patients with visceral fat accumulation may be increasing even though they are only mildly obese, as defined by BMI $(25,26)$. In the present study, we investigated the significance of waist circumference (WC, as an index of visceral fat accumulation), BMI, glycemic control and other clinical parameters on SDB in T2DM to clarify a target for the treatment of SDB and prevention of potential cardiovascular diseases in these patients.

\section{Patients and Methods}

\section{Patients}

One hundred nineteen subjects February 2009 to January 2010 (64 men and 55 women; age $60.6 \pm 1.3$ years, mean \pm SEM, range 24-89, BMI $27.2 \pm 0.6 \mathrm{~kg} / \mathrm{m}^{2}$, range 17.9-52.0) were admitted to the Department of Metabolic Medicine, Osaka University Hospital, for glycemic control during the period from February 2009 to January 2010. Among them, the study subjects were 40 consecutive Japanese patients with T2DM who gave consent and underwent all-night cardiorespiratory monitoring for the present study (21 men and 19 women; age 56.6 \pm 2.2 years, mean \pm SEM, range 30-79). T2DM was defined according to the World Health Organization criteria, and/or treatment for diabetes mellitus. The present study was approved by the ethics committee of Osaka University and a written informed consent was obtained from each participant. [The registration number of the trial at the UMIN is 000002998 (The Endocrine-Metabolic Disease and Sleep Apnea Syndrome Study). https://upload. umin.ac.jp/cgi-open-bin/ctr/ctr.cgi?function=brows\&action= brows\&type $=$ summary \&recptno $=$ R000003635\&language $=E]$

\section{Study protocol}

Each participant underwent all-night cardiorespiratory monitoring from 9:00 PM within 3 days after admission. Af- ter hospitalization, all diabetic patients were treated by oral hypoglycemic agents or insulin injection, or diet alone, for at least one week until fasting plasma glucose level fell below $140 \mathrm{mg} / \mathrm{dL}$. Diet was adjusted by calorie intake per day [25 kcalxideal body weight $(\mathrm{kg}): 22 \times$ height $\left.(\mathrm{m})^{2}\right]$, and patients were encouraged to exercise (about 10,000 steps per day). A second all-night cardiorespiratory monitoring was subsequently performed at $19.6 \pm 1.5$ days after the first study (range 8-41 days). Venous blood samples were collected after overnight fasting in the morning after the first and second all-night monitoring. Excessive daytime sleepiness was assessed using the Epworth Sleepiness Scale (ESS) (27) within 3 days after admission.

\section{Cardiorespiratory monitoring}

Cardiorespiratory monitoring (Somté, Compumedics, Melbourne, Australia) included a pressure sensor for nasal airflow, 2 stress-sensitive belts (for rib cage and abdomen), a body position sensor, pulse oximetry, and electrocardiograph from $9 \mathrm{PM}$ to $6 \mathrm{AM}$ to assess the severity of $\mathrm{SDB}$, as reported previously (12). The recorded signals were analyzed for the number of apneas and hypopneas (28). The duration of sleep was estimated using the self-reported sleep time and the recording data. The oxygen desaturation index (ODI), lowest oxygen saturation, average oxygen saturation, and time at desaturation $<90 \%$ in minutes of total bedtime for the entire night were measured. Apnea represented a decrease in the amplitude of airflow signal or thoracoabdominal motion to $<10 \%$ of the baseline for at least 10 seconds. Hypopnea was defined as a decrease in airflow or respiratory effort to $<70 \%$ of baseline for at least 10 seconds associated with $>4 \%$ desaturation but did not meet the criteria for apnea. The apnea-hypopnea index (AHI) was defined as the total number of apneas/hypopneas per hour of sleep time (29). An AHI $\geq 5$ established the diagnosis of SDB. All recordings were scored manually by an experienced polysomnographer as described in detail previously (12).

We analyzed the low frequency band (LF: 0.04 to 0.15 $\mathrm{Hz}$ ), which is influenced by sympathetic activity, and on the high frequency component (HF: 0.15 to $0.40 \mathrm{~Hz}$ ), which is under vagal control, is synchronous with respiratory frequency, and represents vagal heart activity (30). The LF/HF ratio reflects sympathovagal modulation. Because the total frequency includes the sum of very low, low and high frequency power, the total frequency values were not included in this analysis.

\section{Anthropometry and laboratory measurements}

Height, weight, neck circumference and WC were measured in standing position. Neck circumference at the level of the cricothyroid cartilage and WC at the umbilical level were measured with a non-stretchable tape in late expiration while standing (in $\mathrm{cm}$ ). BMI was calculated [=weight $(\mathrm{kg})$ / height $\left.(\mathrm{m})^{2}\right]$. Blood pressure was measured with a standard mercury sphygmomanometer on the right arm after the subjects had rested in a sitting position for at least 10 minutes. 
Hypertension was defined as systolic blood pressure $\geq 140$ $\mathrm{mmHg}$, diastolic blood pressure $\geq 90 \mathrm{mmHg}$, or treatment for hypertension. Patients with a previous diagnosis of dyslipidemia and hypertension who were on medications for any of these conditions were included in this study. Dyslipidemia was defined as low-density lipoprotein-cholesterol (LDL-C) concentration of $>140 \mathrm{mg} / \mathrm{dL}$, triglyceride (TG) concentration $>150 \mathrm{mg} / \mathrm{dL}$, high-density lipoprotein-cholesterol (HDL-C) concentration $<40 \mathrm{mg} / \mathrm{dL}$, and/or treatment for dyslipidemia. Metabolic syndrome was defined based on the published criteria of the metabolic syndrome for the Japanese population (31). Diabetic retinopathy was assessed by an ophthalmologist. Patients with overt diabetic nephropathy represented those with urinary albumin of $>300 \mathrm{mg} /$ day. Diabetic peripheral neuropathy represented the presence of two of the following three clinical findings: 1) subjective symptoms in lower extremities (pain, numbness, itchiness, coldness, warmness, and weakness), 2) reduction or absence of deep tendon reflex in the both Achilles tendons, and 3) loss of vibration sensation in both lower extremities. Loss of vibration sensation was diagnosed when the duration of vibration sensation was $<10$ seconds using $128-\mathrm{Hz}$ tuning fork placed on the medial malleolus.

Venous blood samples were collected in the morning after overnight fast for measurements of hemoglobin, creatinine, hemoglobin A1c (HbA1c) (Japan Diabetes Society; JDS), total cholesterol, HDL-C, TG, glycoalbumin, 1,5-anhydro-Dglucitol (1,5-AG), glucose, immunoreactive insulin (IRI), CPR (C peptide immunoreactivity). LDL-C was calculated using the Friedewald formula. For the purpose of the present study, blood samples that were obtained from each patient and stored at $-20^{\circ} \mathrm{C}$, were thawed and assayed for adiponectin (Adiponectin ELISA Kit, Otsuka Pharmaceutical Co., Tokushima, Japan) (32), leptin (Human Leptin RIA kit, Linco Research, St. Louis, MO), interleukin 6 (IL6) (QuantiGlo Human IL-6 Immunoassay, R\&D Systems, Minneapolis, MN), monocyte chemotactic protein-1 (MCP1) (Quantikine Human CCL2/MCP-1 Immunoassay, R\&D Systems), high-sensitivity C-reactive protein (hs-CRP) (N-Latex CRP II, Dade Behring Inc, Marburg, Germany), thiobarbituric acid reactive substances (TBARS) (Japan Institute for the Control of Aging, Nikken SEIL Co., Shizuoka, Japan), and brain natriuretic peptide (BNP) (MI02 Shionogi BNP (Human BNP Kit), Shionogi Co., Osaka, Japan). The homeostasis model assessment of insulin resistance (HOMA-IR) was calculated using the following formula: HOMA-IR=[(fasting IRI $\mathrm{mU} / \mathrm{L} \times$ fasting glucose $\mathrm{mg} / \mathrm{dL}$ )/405]. Urinary $\mathrm{CPR}$ was measured in urine collected over a 24-hour period. Patients on medications known to increase serum adiponectin levels, such as pioglitazone (33), and patients with renal dysfunction (creatinine $>1.5 \mathrm{mg} / \mathrm{dL}$ ) were excluded from this study, because renal dysfunction is reported to alter serum adiponectin level (34).

\section{Statistical analysis}

Data are presented as mean \pm SEM. Data of $1,5-\mathrm{AG}$ and
hs-CRP levels showed skewed distribution and were thus log-transformed before analysis. Differences between two groups were compared by Student's t-test or Mann-Whitney U-test. Relationships between two continuous variables were analyzed using scatter plots (Fig. 1) and Pearson's correlation coefficient. Stepwise multiple regression analysis was conducted to identify those parameters that significantly contributed to AHI. Parameters with a $\mathrm{p}$ value of $<0.05$ were subsequently entered into the regression analysis as independent variables. In all cases, 2-tailed $p$ values were used and $\mathrm{p}$ values of $<0.05$ were considered statistically significant. All analyses were performed with the JMP 8 (SAS Institute, Cary, NC).

\section{Results}

\section{Characteristics of patients $(n=40)$}

Table 1, 2 summarize the characteristics of the patients enrolled in this study. The frequency of SDB in patients with T2DM was $90 \%$, similar to the results reported in our previous study in a different group of patients hospitalized for poorly controlled T2DM (12). The average score of ESS was 10.4 for the group. The frequency of sleep apnea syndrome with excessive daytime sleepiness (ESS $\geq 11$ ) among the patients with T2DM was $40 \%(n=16 / 40)$.

\section{Relationship between $\mathrm{AHI}$ and various parameters $(n=40)$}

Table 3 lists the correlation coefficients for the relationship between AHI and various parameters. AHI, determined at the first cardiorespiratory study, correlated positively with BMI, neck circumference, WC, complications of the metabolic syndrome, and systolic and diastolic blood pressures, and tended to correlate with serum $\log$ hs-CRP $(\mathrm{p}=0.05)$ and leptin $(\mathrm{p}=0.05)$. However, there was no correlation between AHI and the duration of DM, ESS scores, 1,5-AG (Table 3), HbA1c and glycoalbumin ( $\mathrm{p}=0.59, \mathrm{p}=0.26$, respectively, data not shown). Stepwise multiple regression analysis identified BMI (multivariate BMI) and WC (multivariate WC) as significant determinants of $\mathrm{AHI}$, whereas 1,5-AG, presence or absence of autonomic disturbance, neck circumference, complications of the metabolic syndrome were not significant (Table 3).

\section{Parameters related to changes in AHI after T2DM therapy $(n=31)$}

Next, 31 patients (17 males and 14 females; 57.9 \pm 2.2 years) were included in a sub-study to investigate the parameters related to changes in AHI recorded at admission and before discharge (duration of hospitalization, average 19.6 days, range $8-41$, median \pm interquartile range $17 \pm 13$ ). The other 9 patients were excluded from the study because of early discharge. Although patients were diagnosed with SDB, they were treated for T2DM but not with nasal continuous positive airway pressure. The individual data are 
Table 1. Baseline Characteristics of All Diabetic Subjects in the Present Study

\begin{tabular}{|c|c|c|}
\hline Age, years & $56.6 \pm 2.2$ & $(30-79)$ \\
\hline Gender (males/females) & $\mathrm{n}=40(21 / 19)$ & \\
\hline Body weight, kg & $78.2 \pm 3.3$ & $(43-138)$ \\
\hline $\mathrm{BMI}, \mathrm{kg} / \mathrm{m}^{2}$ & $29.1 \pm 1.1$ & $(17.9-50.0)$ \\
\hline Neck circumference, $\mathrm{cm}$ & $40.5 \pm 0.5$ & $(35-45)$ \\
\hline WC, cm (males) & $95.0 \pm 3.0$ & $(71-131)$ \\
\hline (females) & $105.4 \pm 4.5$ & $(77-134)$ \\
\hline Smoking (none/ex-smoker/current-smoker) & $n=7 / 17 / 16$ & \\
\hline Duration of DM, years & $11.8 \pm 1.4$ & $(0-30)$ \\
\hline DM neuropathy & $n=13$ & \\
\hline DM retinopathy (NDR/SDR/PDR) & $n=26 / 8 / 6$ & \\
\hline DM nephropathy & $n=11$ & \\
\hline Mets & $n=29$ & \\
\hline Hypertension & $n=24$ & \\
\hline Dyslipidemia & $n=28$ & \\
\hline \multicolumn{3}{|l|}{ Past history } \\
\hline Coronary artery disease & $n=3$ & \\
\hline Cerebrovascular disease & $\mathrm{n}=1$ & \\
\hline Medications for DM & $n=13 / 14 / 8 / 15$ & \\
\hline Medications for HT & $s=12 / 13 / 3 / 6 / 3$ & \\
\hline Epworth sleepiness scale & $10.4 \pm 0.9$ & $(1-21)$ \\
\hline Duration of hospitalization, days & $14.9 \pm 1.5$ & $(2-41)$ \\
\hline \multicolumn{3}{|l|}{ Severity of SDB } \\
\hline No SDB $(\mathrm{AHI} \leq 5)$ & $n=4$ & \\
\hline Mild $(5<\mathrm{AHI} \leq 15)$ & $n=12$ & \\
\hline Moderate $(15<\mathrm{AHI} \leq 30)$ & $n=10$ & \\
\hline Severe $(A H I>30)$ & $n=14$ & \\
\hline AverageSpO ${ }_{2}, \%$ & $94.0 \pm 0.5$ & $(81.0-97.0)$ \\
\hline Lowest $\mathrm{SpO}_{2}, \%$ & $78.6 \pm 1.7$ & $(52.0-91.0)$ \\
\hline $3 \%$ ODI, events/hour & $27.1 \pm 3.7$ & $(0.4-114.4)$ \\
\hline $4 \%$ ODI, events/hour & $20.4 \pm 3.6$ & $(0.6-96.5)$ \\
\hline$\%<90 \%$ time & $9.2 \pm 2.9$ & $(0.1-80.1)$ \\
\hline LF(low frequency)/HF(high frequency) ratio & $2.2 \pm 0.3$ & \\
\hline
\end{tabular}

Mean \pm SEM (range). Numbers $(\mathrm{N})$ of subjects analyzed are shown in parenthesis. BMI; body mass index, WC; waist circumference, DM; diabetes mellitus, NDR: non diabetic retinopathy, SDR; simple diabetic retinopathy, PDR; proliferarive diabetic retinopathy, Mets; the metabolic syndrome, SU; sulfonyl urea, BG; biguanide, $\alpha \mathrm{Gl}$; alpha glucosidase inhibitor, HT; hypertension, $\mathrm{CA}$; calcium channel antagonist, $\mathrm{ACEl}$; angiotensin converting enzyme inhibitor, ARB; angiotensin receptor blocker, $\beta ; \beta$ blockade, SDB; sleep-disordered breathing, , $\mathrm{AHI}$; apnea-hypopnea index, $\mathrm{SpO}_{2}$; percentage of arterial $\mathrm{O}_{2}$ saturation from pulse oximetry, ODI; oxygen desaturation index, $90 \%<$ time; time at desaturation below $90 \%$ in minutes of total bedtime

shown in Table 4. There were significant reductions in BMI, WC, fasting glucose, 1,5-AG, glycoalbumin, HbA1c, LDL$\mathrm{C}$, serum leptin, hs-CRP, and TBARS levels. The AHI was lower at discharge than admission. There were no significant changes in serum adiponectin, IL-6, and plasma MCP1 levels during hospitalization.

We investigated the relationships between changes in AHI and several parameters, which showed significant changes at discharge (Table 4). The $\triangle \mathrm{AHI}$ correlated positively with $\Delta \mathrm{WC}(\mathrm{r}=0.53, \mathrm{p}<0.01$, Fig. 1B), but not with $\Delta \mathrm{BMI}$ (Fig. 1A) or $\Delta 1,5-\mathrm{AG}$ (Fig. 1C). Furthermore, $\Delta$ AHI was not correlated with changes in serum adiponectin, leptin, hsCRP or TBARS (data not shown).

\section{Discussion}

The present study showed that WC and BMI were correlated with AHI independent of glucose levels and neuropathy in patients with T2DM. More importantly, the extent of
WC reduction correlated with improvement in SDB independent of glycemic control after short-term hospitalization for treatment of T2DM.

Recently, obesity has been considered as a trigger of a variety of lifestyle-related disorders such as T2DM, dyslipidemia, hypertension, hyperuricemia, non-alcoholic steatohepatitis, chronic kidney disease, sleep apnea-hypopnea syndrome and life-threatening atherosclerotic cardiovascular diseases (35-37). Substantial evidence demonstrated that the association of these disorders in obesity is related to body fat distribution rather than absolute body weight (17, 38-44). Accumulation of intra-abdominal visceral fat significantly contributes to the association of these disorders (45). WC is widely-used as a conventional index for excess visceral fat and/or abdominal obesity. In East and South Asian countries, people have a variety of lifestyle-related disorders despite relatively lower BMI compared to Caucasians, suggesting the importance of excess visceral fat in these populations $(46,47)$. 
Table 2. Laboratory Data of All Diabetic Subjects in the Present Study

\begin{tabular}{lrl}
\hline $\mathrm{Hb}, \mathrm{g} / \mathrm{dL}$ & $13.9 \pm 0.2$ & $(11.1-16.5)$ \\
Creatinine, mg/dL & $0.80 \pm 0.09$ & $(0.45-1.06)$ \\
Fasting Glucose, mg/dL & $174 \pm 9$ & $(81-325)$ \\
s-CPR, mg/dL & $2.1 \pm 0.2$ & $(0.4-5.2)$ \\
$1,5-\mathrm{AG}, \mu \mathrm{g} / \mathrm{mL}$ & $6.3 \pm 1.0$ & $(1.0-24.5)$ \\
$\mathrm{HbA} 1 \mathrm{c}(\mathrm{JDS}), \%$ & $9.0 \pm 0.4$ & $(5.2-16.8)$ \\
Glycoalbumin, \% & $24.2 \pm 1.5$ & $(11.2-47.1)$ \\
HOMA-IR, units, N=22 & $2.65 \pm 0.41$ & $(0.5-7.85)$ \\
$\mathrm{u}-\mathrm{CPR}, \mu \mathrm{g} / \mathrm{day}$ & $56 \pm 8$ & $(5.1-216)$ \\
T-cho, mg/dL & $202 \pm 6$ & $(127-287)$ \\
LDL-C, mg/dL & $125 \pm 6$ & $(68-208)$ \\
TG, mg/dL & $161 \pm 15$ & $(52-549)$ \\
HDL-C, mg/dL (all) & $46 \pm 2$ & $(32-71)$ \\
HDL-C, mg/dL (males) & $45 \pm 2$ & $(32-66)$ \\
HDL-C, mg/dL (females) & $49 \pm 2$ & $(36-71)$ \\
serum adiponectin, $\mu \mathrm{g} / \mathrm{mL}$ (all) & $7.1 \pm 0.8$ & $(2.5-26.37)$ \\
adiponectin, $\mu \mathrm{gg} / \mathrm{mL}$ (males) & $8.0 \pm 1.4$ & $(2.5-26.37)$ \\
adiponectin, $\mu \mathrm{gg} / \mathrm{mL}$ (females) & $6.1 \pm 0.7$ & $(2.91-14.97)$ \\
serum Leptin, $\mathrm{ng} / \mathrm{mL}$ & $11.4 \pm 1.2$ & $(2.1-28.6)$ \\
serum IL6, pg/mL & $2.6 \pm 0.6$ & $(0.3-12.6)$ \\
plasma MCP1, pg/mL & $161.9 \pm 6.9$ & $(12.8-232.2)$ \\
serum hs-CRP, mg/dL & $0.181 \pm 0.028$ & $(0.018-0.295)$ \\
serum TBARS, nmol/mL & $4.4 \pm 0.1$ & $(3.1-6.0)$ \\
plasma BNP, pg/mL & $27.6 \pm 16.3$ & $(4-43.9)$ \\
\hline
\end{tabular}

Mean \pm SEM (range). Numbers $(\mathrm{N})$ of subjects analyzed are shown in parenthesis. $\mathrm{Hb}$; hemoglobin, s-CPR; serum $\mathrm{C}$ peptide, 1,5-AG; 1,5anhydro-D-glucitol, HOMA-IR; homeostasis model assessment-insulin resistance , u-CPR; urinary $\mathrm{C}$ peptide, T-cho; total cholesterol, LDL-C; low density lipoprotein-cholesterol, TG; triglyceride, HDL-C; high density lipoprotein-cholesterol, IL6; interleukin 6, MCP1, monocyte chemotactic protein-1, hs-CRP; high-sensitivity C-reactive protein, TBARS; thiobarbituric acid reactive substances, BNP; brain natriuretic peptide

We reported previously that hospitalized T2DM patients frequently have SDB (12). In the present study, we found that WC was correlated with AHI independent of BMI (Table 3). Furthermore, improvement in AHI correlated significantly with reduction of WC, but not in BMI (Fig. 1). These findings indicate that excess intraabdominal fat is more important than absolute body weight for the association of SDB even though the patients had mild obesity.

The hypothesis that hyperglycemia per se plays an important role in the development of SDB is fascinating. It is also interesting that functional disturbance of the autonomic nervous system associated with diabetic neuropathy may worsen the regulation of breathing. However, we could not find any significant relationship between AHI and glycemic control or presence of diabetic neuropathy. Further studies on the effect of long-term control of blood glucose level or evaluation of autonomic nervous system activity will be necessary in the future.

The adipose tissue, i.e., masses of adipocytes, is present in many areas of the human body. Adipocytes produce and secrete a variety of bioactive substances called adipocytokines (48). However, accumulation of visceral fat results in adipocyte dysfunction (49), infiltration of macrophages into the adipose tissue (50-52), and the production of reactive oxygen species locally in the adipose tissue (21). Under such an environment, serum levels of hs-CRP, systemic oxidative stress (53), and leptin are elevated, whereas adiponectin, an adipocytokine with anti-inflammatory, antidiabetic and anti-atherogenic properties, is decreased in subjects with visceral fat accumulation (54). In the present study, the levels of circulating leptin, adiponectin, and IL6 on admission were significantly correlated with BMI $(r=$ $0.68, \mathrm{p}<0.01 ; \mathrm{r}=-0.42, \mathrm{p}<0.01 ; \mathrm{r}=0.43, \mathrm{p}<0.01$; respectively, data not shown), and $\mathrm{WC}(\mathrm{r}=0.70, \mathrm{p}<0.01 ; \mathrm{r}=-0.42, \mathrm{p}<0.01$; $\mathrm{r}=0.39, \mathrm{p}<0.05$; respectively, data not shown), but circulating MCP1 levels were not correlated. The percent changes in these parameters, particularly leptin, between admission and discharge were significantly correlated with $\triangle \mathrm{BMI}(\mathrm{r}=0.44$, $\mathrm{p}<0.05)$ and $\Delta \mathrm{WC}(\mathrm{r}=0.36, \mathrm{p}<0.05)($ data not shown). Taken together, dysregulated production of cytokines in T2DM patients with SDB may relate to poor glycemic control with weight gain, rather than SDB. SDB and dysregulation of adipocyte-derived factors in subjects with abdominal obesity may independently and synergistically contribute to the development of cardiovascular diseases. Further long-term study should be conducted in the future.

In conclusion, we described here that reduction of WC was correlated with improvement in SDB in Japanese T2DM patients, suggesting that any program designed to prevent cardiovascular disease in diabetic patients should include continuous evaluation of SDB and WC. Longer observational and interventional trials should be conducted to assess the effect of WC reduction on the incidence of cardiovascular events in diabetic patients with SDB.

\section{Study limitations}

Several limitations of this study must be considered. First, we assessed the severity of SDB in 40 hospitalized patients with T2DM by all-night cardiorespiratory monitoring on two occasions (within 3 days after admission and at 19.6 \pm 1.5 days after the first study) during intensive T2DM treatment. However, the number of patients may be still small, and further investigations might be required to confirm the present results. Also, there might be a selection bias for the severity of T2DM since they were the ones who required hospital admission. The patients might have relatively higher BMI and larger WC compared to the average physical constitution of Japanese patients with T2DM. Second, all patients in this study were Japanese and any differences from other ethnicities are unknown. Third, although patients were diagnosed with SDB, they were treated for T2DM but not with nasal continuous positive airway pressure, which is the golden standard treatment of obstructive sleep apnea. It is because the aim of this study was to compare the effect of glycemic control on changes in SDB and SDB-related parameters from admission to discharge after anti-diabetes therapy in patients hospitalized with T2DM. Fourth, the current uncontrolled study demonstrated that changes in AHI did not correlate with changes in $1,5-\mathrm{AG}$, possibly because 
Table 3. Correlation between AHI and Various Clinico-biochemical Parameters and Results of Stepwise Multiple Regression Analysis for AHI

\begin{tabular}{|c|c|c|c|c|}
\hline \multirow{3}{*}{$\mathrm{AHI}$} & \multirow{2}{*}{\multicolumn{2}{|c|}{ Univariate }} & \multicolumn{2}{|c|}{ Multivariate } \\
\hline & & & \multirow{2}{*}{$\frac{(\mathrm{BMI})}{\mathrm{p} \text { value }}$} & \multirow{2}{*}{$\frac{(\mathrm{WC})}{\mathrm{p} \text { value }}$} \\
\hline & $r$ & $p$ value* & & \\
\hline Age & -0.27 & 0.09 & & \\
\hline Duration of DM & -0.05 & 0.75 & & \\
\hline $\mathrm{BMI}$ & 0.66 & $<0.01$ & $<0.01$ & $\longrightarrow$ \\
\hline Neck circumference & 0.51 & $<0.01$ & 0.66 & 0.55 \\
\hline WC (all) & 0.66 & $<0.01$ & 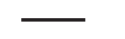 & $<0.01$ \\
\hline (males) & 0.65 & $<0.01$ & & \\
\hline (females) & 0.72 & $<0.01$ & & \\
\hline Complication of Mets & & $<0.05$ & 0.44 & 0.39 \\
\hline LF/HF ratio & 0.19 & 0.24 & & \\
\hline Systolic blood pressure & 0.41 & $<0.01$ & 0.06 & 0.11 \\
\hline Diastolic blood pressure & 0.37 & $<0.05$ & & \\
\hline Epworth score scale & 0.05 & 0.75 & & \\
\hline $\log 1,5-A G$ & 0.01 & 0.93 & & \\
\hline adiponectin (all) & -0.03 & 0.87 & & \\
\hline Leptin & 0.31 & 0.05 & & \\
\hline IL6 & 0.28 & 0.08 & & \\
\hline MCP1 & 0.01 & 0.60 & & \\
\hline Log hs-CRP & 0.28 & 0.08 & & \\
\hline TBARS & -0.06 & 0.71 & & \\
\hline \multirow[t]{2}{*}{ BNP } & 0.15 & 0.35 & & \\
\hline & & & $\begin{array}{l}\text { Adjusted } \\
r=0.67\end{array}$ & $\begin{array}{l}\text { Adjusted } \\
r=0.67\end{array}$ \\
\hline
\end{tabular}

Mets; the metabolic syndrome based on the Japanese criteria.

* by Pearson's correlation analysis. Stepwise multiple regression analysis was conducted to identify those parameters that significantly contributed to $\mathrm{AHI}$ (adopted factors; Multivariate (BMI); BMI, neck circumference, complication of Mets, systolic blood pressure, Multivariate (WC); , neck circumference, WC, complication of Mets, systolic blood pressure). Parameters with $P$ value $<0.05$ were subsequently entered into the regression analysis as independent variables.

the glycemic level improved in all participants by intensive glucose lowering therapy. Further controlled studies of blind and randomized design are required. Fifth, although we used WC to evaluate visceral fat accumulation in the present study, research on both visceral and subcutaneous fat area measured by computed tomography scan is needed to clarify the effect of visceral and subcutaneous adiposity on AHI. Finally, we screened for SDB using type 3 cardiopulmonary monitoring. All recordings were subsequently scored manually by an experienced registered polysomnographer, using a simplified cardiorespiratory monitoring device (28). Devices without the electroencephalogram cannot record the sleep stage. Further investigation using a full polysomnography might be required to confirm the present results.

The authors state that they have no Conflict of Interest (COI).

\section{Acknowledgement}

We are grateful to Ikuo Nagata and Hiroshi Kanatani for the statistical advice, and Masato Tanaka and Misato Nakano for help with apnomonitor scoring and analysis. This work was supported in part by a Grant-in-Aid for Scientific Research No. (C) No. 21591177 (to K.K.).

\section{References}

1. Findley LJ, Fabrizio M, Thommi G, Suratt PM. Severity of sleep apnea and automobile crashes. N Engl J Med 320: 868-869, 1989.

2. Gami AS, Howard DE, Olson EJ, Somers VK. Day-night pattern of sudden death in obstructive sleep apnea. N Engl J Med 342: 1206-1214, 2005.

3. Marin JM, Carrizo SJ, Vicente E, Agusti AG. Long-term cardiovascular outcomes in men with obstructive sleep apnoeahypopnoea with or without treatment with continuous positive airway pressure: an observational study. Lancet 365: 1046-1053, 2005.

4. Katsumata K, Okada T, Miyao M, Katsumata Y. High incidence of sleep apnea syndrome in a male diabetic population. Diabetes Res Clin Pract 13: 45-51, 1991.

5. Sridhar GR, Madhu K. Prevalence of sleep disturbances in diabetes mellitus. Diabetes Res Clin Pract 23: 183-186, 1994. 
Table 4. Changes in Various Parameters Induced by the Treatment Protocol $(n=31)$

\begin{tabular}{|c|c|c|c|}
\hline & Admission & Discharge & $p$ value \\
\hline Body weight, kg & $76.4 \pm 3.5$ & $73.8 \pm 3.3$ & $<0.01$ \\
\hline BMI, kg/m² & $28.5 \pm 1.2$ & $27.3 \pm 1.1$ & $<0.05$ \\
\hline Waist circumferance, cm (all) & $97.5 \pm 2.6$ & $95.6 \pm 2.5$ & $<0.05$ \\
\hline Waist circumferance, $\mathrm{cm}$ (males) & $93.6 \pm 3.2$ & $92.2 \pm 3.4$ & $<0.05$ \\
\hline Waist circumferance, $\mathrm{cm}$ (females) & $104.3 \pm 4.3$ & $102.8 \pm 4.1$ & $<0.05$ \\
\hline Fasting Glucose, mg/dL & $176 \pm 11$ & $123 \pm 5$ & $<0.01$ \\
\hline 1,5-AG, $\mu \mathrm{g} / \mathrm{mL}$ & $5.9 \pm 1.1$ & $8.3 \pm 0.9$ & $<0.05$ \\
\hline Glycoalbumin, \% & $26.4 \pm 1.5$ & $20.4 \pm 1.0$ & $<0.01$ \\
\hline HbA1c (JDS), \% & $9.1 \pm 1.1$ & $8.3 \pm 0.4$ & $<0.01$ \\
\hline adiponectin, $\mu \mathrm{g} / \mathrm{mL}$ (all) & $6.7 \pm 1.0$ & $6.7 \pm 1.2$ & 0.50 \\
\hline adiponectin, $\mu \mathrm{g} / \mathrm{mL}$ (males) & $7.8 \pm 1.5$ & $7.5 \pm 1.7$ & 0.52 \\
\hline adiponectin, $\mu \mathrm{g} / \mathrm{mL}$ (females) & $5.4 \pm 0.6$ & $5.4 \pm 0.5$ & 0.91 \\
\hline $\mathrm{Hs}-\mathrm{CRP}, \mathrm{mg} / \mathrm{dL}$ & $0.21 \pm 0.03$ & $0.15 \pm 0.03$ & $<0.05$ \\
\hline TBARS, $\mathrm{nmol} / \mathrm{mL}$ & $4.45 \pm 0.12$ & $3.94 \pm 0.12$ & $<0.01$ \\
\hline BNP, pg/mL & $12.7 \pm 2.6$ & $13.4 \pm 2.5$ & 0.71 \\
\hline $\mathrm{s}-\mathrm{CPR}, \mathrm{mg} / \mathrm{dL}$ & $2.1 \pm 0.2$ & $1.8 \pm 0.3$ & 0.92 \\
\hline Leptin, ng/mL & $12.0 \pm 1.5$ & $9.5 \pm 1.2$ & $<0.01$ \\
\hline IL6, pg/mL & $2.5 \pm 0.6$ & $1.5 \pm 0.3$ & 0.12 \\
\hline $\mathrm{MCP} 1, \mathrm{pg} / \mathrm{mL}$ & $210.7 \pm 44.6$ & $213.4 \pm 44.3$ & 0.65 \\
\hline LDL-C, mg/dL & $127 \pm 7$ & $103 \pm 5$ & $<0.01$ \\
\hline HDL-C, mg/dL & $47 \pm 2$ & $46 \pm 2$ & 0.09 \\
\hline $\mathrm{TG}, \mathrm{mg} / \mathrm{dL}$ & $164 \pm 18$ & $113 \pm 8$ & $<0.05$ \\
\hline AHI, events/hour & $25.6 \pm 4.3$ & $21.1 \pm 3.9$ & $<0.01$ \\
\hline AverageSpO ${ }_{2}, \%$ & $94.1 \pm 0.5$ & $94.9 \pm 0.5$ & $<0.01$ \\
\hline Lowest $\mathrm{SpO}_{2}, \%$ & $77.9 \pm 2.0$ & $80.1 \pm 1.4$ & 0.12 \\
\hline $3 \%$ ODI, events/hour & $28.5 \pm 3.8$ & $22.4 \pm 3.7$ & $<0.05$ \\
\hline $4 \% O D I$, events/hour & $21.7 \pm 3.7$ & $16.5 \pm 3.4$ & $<0.05$ \\
\hline$\%<90 \%$ time & $9.6 \pm 3.6$ & $6.5 \pm 2.9$ & $<0.05$ \\
\hline OSAI, events/hour & $8.9 \pm 3.0$ & $6.6 \pm 2.7$ & $<0.05$ \\
\hline CSAI, events/hour & $2.6 \pm 0.9$ & $2.3 \pm 1.1$ & 0.53 \\
\hline Hypopnea index, events/hour & $15.0 \pm 2.4$ & $12.1 \pm 2.6$ & $<0.05$ \\
\hline Mixed apnea index, events/hour & $1.1 \pm 0.4$ & $0.7 \pm 0.3$ & 0.09 \\
\hline Duration of hospitalization, days & & $19.6 \pm 1.5$ & \\
\hline Use of insulin injection, \% & $n=9 / 31(29 \%)$ & $n=21 / 31(68 \%)$ & \\
\hline
\end{tabular}

Mean \pm SEM. AHI; apnea-hypopnea index, $\mathrm{SpO}_{2}$; percentage of arterial $\mathrm{O}_{2}$ saturation, ODI; oxygen desaturation index, $90 \%<$ time; time at desaturation below $90 \%$ in minutes of total bedtime, OSAI; obstructive seep apnea index, CSAI; central sleep apnea index

A

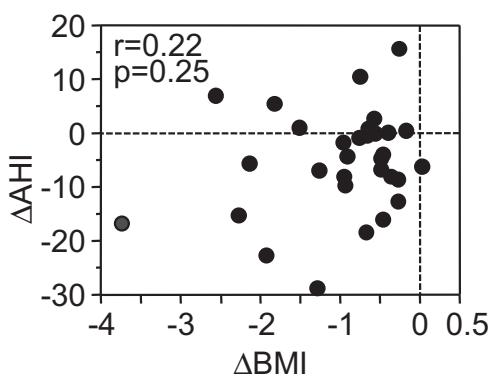

B

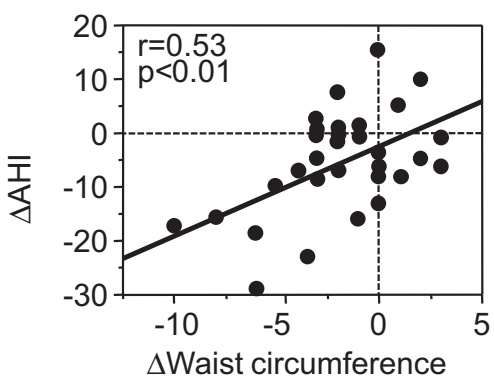

C

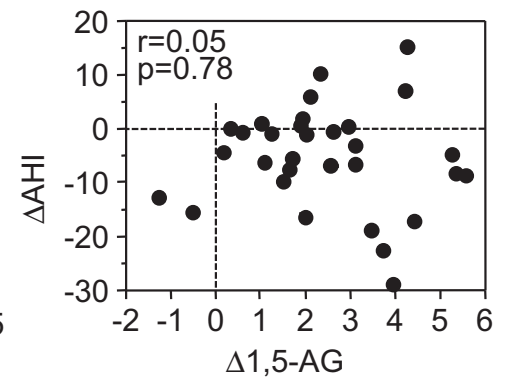

Figure 1. Relationships between $\Delta \mathrm{BMI}, \Delta \mathrm{WC}, \Delta 1,5-\mathrm{AG}$ and $\Delta \mathrm{AHI}$. Pearson's correlation coefficient was used to examine the relationship between changes in the apnea-hypopnea index (AHI) $(\triangle \mathrm{AHI})$ and each parameter measured on admission and before discharge. A: $\Delta$ body mass index (BMI), B: $\Delta$ waist circumference (WC), C: $\Delta 1,5$-anhydro-D-glucitol (1,5-AG). 
6. Resnick HE, Redline S, Shahar E, et al; Sleep Heart Health Study. Diabetes and sleep disturbances: findings from the Sleep Heart Health Study. Diabetes Care 26: 702-709, 2003.

7. Reichmuth KJ, Austin D, Skatrud JB, Young T. Association of sleep apnea and type II diabetes: a population-based study. Am J Respir Crit Care Med 172: 1590-1595, 2005.

8. Einhorn D, Stewart DA, Erman MK, Gordon N, Philis-Tsimikas A, Casal E. Prevalence of sleep apnea in a population of adults with type 2 diabetes mellitus. Endocr Pract 13: 355-362, 2007.

9. Pascualy R. Sleep apnea and type 2 diabetes. A vicious circle. Diabetes Self Manag 24: 29-30, 2007.

10. Martins RC, Andersen ML, Tufik S. The reciprocal interaction between sleep and type 2 diabetes mellitus: facts and perspectives. Braz J Med Biol Res 4: 180-187, 2008.

11. Tasali E, Mokhlesi B, Van Cauter E. Obstructive sleep apnea and type 2 diabetes: interacting epidemics. Chest 133: 496-506, 2008.

12. Kashine S, Kishida K, Funahashi T, et al. Characteristics of sleepdisordered breathing in Japanese patients with type 2 diabetes mellitus. Metabolism 59: 690-696, 2010.

13. Shamsuzzaman AS, Gersh BJ, Somers VK. Obstructive sleep apnea: implications for cardiac and vascular disease. JAMA 290: 1906-1914, 2003.

14. Ferini-Strambi L, Fantini ML. Cerebrovascular diseases and sleepdisordered breathing. Clin Exp Hypertens 28: 225-231, 2006.

15. Budhiraja R, Budhiraja P, Quan SF. Sleep-disordered breathing and cardiovascular disorders. Respir Care 55: 1322-1332, 2010.

16. Shaw JE, Punjabi NM, Wilding JP, Alberti KG, Zimmet PZ; International Diabetes Federation Taskforce on Epidemiology and Prevention. Sleep-disordered breathing and type 2 diabetes: a report from the International Diabetes Federation Taskforce on Epidemiology and Prevention. Diabetes Res Clin Pract 81: 2-12, 2008.

17. Shinohara E, Kihara S, Yamashita $S$, et al. Visceral fat accumulation as an important risk factor for obstructive sleep apnoea syndrome in obese subjects. J Intern Med 241: 11-18, 1997.

18. Vgontzas AN, Bixler EO, Chrousos GP. Metabolic disturbances in obesity versus sleep apnoea: the importance of visceral obesity and insulin resistance. J Intern Med 254: 32-44, 2003.

19. Vgontzas AN, Bixler EO, Chrousos GP. Metabolic disturbances in obesity versus sleep apnoea: the importance of visceral obesity and insulin resistance. J Intern Med 254: 32-44, 2003.

20. Kishida K, Funahashi T, Matsuzawa Y, Shimomura I. Visceral adiposity as a target for the management of the metabolic syndrome: A Japanese perspective. Ann Med 2011 in press.

21. Furukawa S, Fujita T, Shimabukuro M, et al. Increased oxidative stress in obesity and its impact on metabolic syndrome. J Clin Invest 114: 1752-1761, 2004.

22. Nakagawa $Y$, Kishida $K$, Kihara $S$, et al. Nocturnal reduction in circulating adiponectin concentrations related to hypoxic stress in severe obstructive sleep apnea-hypopnea syndrome. Am J Physiol Endocrinol Metab 294: E778-E784, 2008.

23. Nakagawa $Y$, Kishida K, Kihara S, Yoshida R, Funahashi T, Shimomura I. Nocturnal falls of adiponectin levels in sleep apnea with abdominal obesity and impact of hypoxia-induced dysregulated adiponectin production in obese murine mesenteric adipose tissue. J Atheroscler Thromb 18: 240-247, 2011.

24. Huxley R, James WP, Barzi F, et al; Obesity in Asia Collaboration. Ethnic comparisons of the cross-sectional relationships between measures of body size with diabetes and hypertension. Obes Rev 9: 53-61, 2008.

25. Anan F, Masaki T, Eto T, et al. Visceral fat accumulation is a significant risk factor for white matter lesions in Japanese type 2 diabetic patients. Eur J Clin Invest 39: 368-374, 2009.

26. Anan F, Masaki T, Umeno Y, et al. Correlations of visceral fat accumulation and atherosclerosis in Japanese patients with type 2 diabetes mellitus. Metabolism 57: 280-284, 2008.

27. Johns MW. A new method for measuring daytime sleepiness: the
Epworth sleepiness scale. Sleep 14: 540-545, 1991.

28. Collop NA, Anderson WM, Boehlecke B, et al; Portable Monitoring Task Force of the American Academy of Sleep Medicine. Clinical guidelines for the use of unattended portable monitors in the diagnosis of obstructive sleep apnea in adult patients. Portable Monitoring Task Force of the American Academy of Sleep Medicine. J Clin Sleep Med 3: 737-747, 2007.

29. Anonymous. Sleep-related breathing disorders in adults: recommendations for syndrome definition and measurement techniques in clinical research. The Report of an American Academy of Sleep Medicine Task Force. Sleep 22: 667-689, 1999.

30. Anonymous. Task force of the European society of cardiology the North American Society of pacing and electrophysiology. Heart rate variability: standards of measurement, physiological interpretation, and clinical use. Circulation 93: 1043-1065, 1996.

31. Teramoto T, Sasaki J, Ueshima H, et al. Metabolic syndrome. J Atheroscler Thromb 15: 1-5, 2008.

32. Arita $\mathrm{Y}$, Kihara $\mathrm{S}$, Ouchi $\mathrm{N}$, et al. Paradoxical decrease of an adipose-specific protein, adiponectin, in obesity. Biochem Biophys Res Commun 257: 79-83, 1999.

33. Maeda N, Takahashi M, Funahashi T, et al. PPAR gamma ligands increase expression and plasma concentrations of adiponectin, an adipose-derived protein. Diabetes 50: 2094-2099, 2001.

34. Zoccali C, Mallamaci F, Tripepi G, et al. Adiponectin, metabolic risk factors, and cardiovascular events among patients with endstage renal disease. J Am Soc Nephrol 13: 134-141, 2002.

35. Roman S, Pandolfino JE. Environmental - lifestyle related factors. Best Pract Res Clin Gastroenterol 24: 847-859, 2010.

36. Egger G, Dixon J. Obesity and chronic disease: always offender or often just accomplice? Br J Nutr 102: 1238-1242, 2009.

37. Dubbert PM, Carithers T, Sumner AE, et al. Obesity, physical inactivity, and risk for cardiovascular disease. Am J Med Sci 324: 116-126, 2002.

38. Fujioka S, Matsuzawa Y, Tokunaga K, Tarui S. Contribution of intra-abdominal fat accumulation to the impairment of glucose and lipid metabolism in human obesity. Metabolism 36: 54-59, 1987.

39. Nakajima T, Fujioka S, Tokunaga K, Matsuzawa Y, Tarui S. Correlation of intraabdominal fat accumulation and left ventricular performance in obesity. Am J Cardiol 64: 369-373, 1989.

40. Kanai H, Matsuzawa Y, Kotani K, et al. Close correlation of intraabdominal fat accumulation to hypertension in obese women. Hypertension 16: 484-490, 1990.

41. Fujioka S, Matsuzawa $Y$, Tokunaga K, et al. Improvement of glucose and lipid metabolism associated with selective reduction of intra-abdominal visceral fat in premenopausal women with visceral fat obesity. Int J Obes 15: 853-859, 1991.

42. Matsuzawa Y, Fujioka S, Tokunaga K, Tarui S. Classification of obesity with respect to morbidity. Proc Soc Exp Biol Med 200: 197-201, 1992.

43. Kanai H, Tokunaga K, Fujioka S, Yamashita S, Kameda-Takemura KK, Matsuzawa Y. Decrease in intra-abdominal visceral fat may reduce blood pressure in obese hypertensive women. Hypertension 27: 125-129, 1996.

44. Matsuzawa Y. Establishment of a concept of visceral fat syndrome and discovery of adiponectin. Proc Jpn Acad Ser B Phys Biol Sci 86: 131-141, 2010.

45. Nakamura T, Tokunaga K, Shimomura I, et al. Contribution of visceral fat accumulation to the development of coronary artery disease in non-obese men. Atherosclerosis 107: 239-246, 1994.

46. Fujimoto WY, Bergstrom RW, Boyko EJ, et al. Visceral adiposity and incident coronary heart disease in Japanese-American men. The 10-year follow-up results of the Seattle Japanese-American Community Diabetes Study. Diabetes Care 22: 1808-1812, 1999.

47. Kadowaki T, Sekikawa A, Murata K, et al. Japanese men have larger areas of visceral adipose tissue than Caucasian men in the same levels of waist circumference in a population-based study. 
Int J Obes (Lond) 30: 1163-1165, 2006.

48. Funahashi T, Nakamura T, Shimomura I, et al. Role of adipocytokines on the pathogenesis of atherosclerosis in visceral obesity. Intern Med 38: 202-206, 1999.

49. Funahashi T, Matsuzawa Y. Metabolic syndrome: clinical concept and molecular basis. Ann Med 39: 482-494, 2007.

50. Suganami T, Nishida J, Ogawa Y. A paracrine loop between adipocytes and macrophages aggravates inflammatory changes: role of free fatty acids and tumor necrosis factor alpha. Arterioscler Thromb Vasc Biol 25: 2062-2068, 2005.

51. Suganami T, Tanimoto-Koyama K, Nishida $J$, et al. Role of the Toll-like receptor 4/NF-kappaB pathway in saturated fatty acid- induced inflammatory changes in the interaction between adipocytes and macrophages. Arterioscler Thromb Vasc Biol 27: 84-91, 2007.

52. Suganami T, Ogawa Y. Adipose tissue macrophages: their role in adipose tissue remodeling. J Leukoc Biol 88: 33-39, 2010.

53. Fujita K, Nishizawa H, Funahashi T, Shimomura I, Shimabukuro M. Systemic oxidative stress is associated with visceral fat accumulation and the metabolic syndrome. Circ J 70: 1437-1442, 2006.

54. Ryo M, Nakamura T, Kihara $\mathrm{S}$, et al. Adiponectin as a biomarker of the metabolic syndrome. Circ J 68: 975-981, 2004.

(C) 2011 The Japanese Society of Internal Medicine http://www.naika.or.jp/imindex.html 